\title{
Wenn es gut werden muss: Projektmanagement für reale Aufgaben
}

\author{
Jörg Longmuß und Benjamin Höhne
}

\begin{tabular}{l|l} 
Steckbrief & \\
\hline Unternehmen & $\begin{array}{l}\text { Bayer Pharma Berlin - Bereich Technik. Der Standort hat über 5000 } \\
\text { Beschäftigte, der Bereich ca. 300 Beschäftigte. }\end{array}$ \\
\hline Auftrag & $\begin{array}{l}\text { Eine praxisintegrierte Kompetenzentwicklung zum Thema Projektmanagement } \\
\text { für erfahrene Mitarbeiter }\end{array}$ \\
\hline Rollen & $\begin{array}{l}\text { Lernende: Mitarbeiter auf dem Niveau Meister/Techniker mit über 10 Jahren } \\
\text { Berufserfahrung } \\
\text { Begleitung: Zwei externe Lernbegleiter, einer für die fachliche Seite des } \\
\text { Projektmanagements, einer für die kommunikative Ebene. Zusätzlich wurden } \\
\text { bei Bedarf unternehmensinterne Experten eingeladen. } \\
\text { Auftraggeber: Übergreifend der Leiter des Bereichs Technik, für die einzelnen } \\
\text { Aufgaben (s. u.) jeweils die Abteilungsleiter. }\end{array}$ \\
\hline Ablauf: & $\begin{array}{l}\text { Die Mitarbeiter lernten mit einem Aufwand von ca. einem Tag pro Woche in } \\
\text { zwei Phasen: } \\
\text { 1. gemeinsam eine Studie zu einer betrieblichen Fragestellung erstellen; } \\
\text { 2. individuell eine reale Aufgabe aus dem eigenen Bereich bearbeiten. } \\
\text { Es gab über sechs Monate meist wöchentliche Treffen mit den Begleitern, bei } \\
\text { Zwischenbilanzen auch mit den Vorgesetzten. Die Lerninhalte zum } \\
\text { Projektmanagement kamen von den externen Begleitern, die fachlichen } \\
\text { Aufgaben von den Vorgesetzten. }\end{array}$ \\
\hline Technik & $\begin{array}{l}\text { Für den Dokumentenaustausch wurde zum Teil ein MS Sharepoint eingesetzt, } \\
\text { für die kurzfristige und formlose Kommunikation ein Messenger Dienst. }\end{array}$ \\
\hline
\end{tabular}

J. Longmuß $(\bowtie)$

SUSTAINUM - Institut für zukunftsfähiges Wirtschaften Berlin, Berlin, Deutschland

E-Mail: j.longmuss@sustainum.de

B. Höhne

Fernstudieninstitut, Beuth Hochschule für Technik Berlin, Berlin, Deutschland

E-Mail: bhoehne@beuth-hochschule.de

(C) Der/die Herausgeber bzw. der/die Autor(en) 2021

J. Longmuß et al. (Hrsg.), Agiles Lernen im Unternehmen,

https://doi.org/10.1007/978-3-662-62013-7_4 


\begin{tabular}{l|l}
\hline Besonderheiten & $\begin{array}{l}\bullet \text { Die Aufgaben im zweiten Teil waren reale Projekte, die z. T. aktuell } \\
\text { durchgeführt werden mussten. } \\
\bullet \text { Die Lerninhalte bewegten sich auf akademischem Niveau. }\end{array}$ \\
\hline
\end{tabular}

\subsection{Vorbereitung}

\section{Was fehlt uns für die nächste Ebene?}

$\mathrm{Zu}$ Beginn des Projekts „Brofessio - Berufliche Professionalität im produzierenden Gewerbe“" (siehe Ahrens et al. 2018) gab es am Standort Berlin einen Einstellungsstopp, so dass auch keine neuen Ingenieure eingestellt werden konnten. Gleichzeitig arbeiteten im Bereich Technik viele hoch qualifizierte Fachleute mit gewerblichem Hintergrund. Die Aufgabe des Lernprojektes war es, diese Mitarbeitergruppe für die Übernahme von Tätigkeiten auf Ingenieurniveau zu qualifizieren.

In einer umfassenden Arbeitsprozess-Analyse über mehrere Monate wurden fünf arbeitsplatzübergreifende Kernarbeitsprozesse für die Ebene der Meister und Techniker identifiziert. Acht Mitarbeiter bewerteten diese nach Schwierigkeit und persönlicher Herausforderung. Abschließend nannten die Befragten entsprechende Arbeitsinhalte, bei denen sie für sich einen Bedarf für Kompetenzentwicklung sahen. Das Ergebnis war eindeutig: Zentraler Bedarf war es, Projekte mit Methoden des Projektmanagements planen und umsetzen zu können (siehe Abb. 4.1).

Am Ende eines langen Auswahlprozesses hatte sich eine Gruppe von vier Beschäftigten etabliert, die sowohl das Interesse als auch die Bereitschaft zur Teilnahme an dem Lernprojekt und zudem innerbetrieblich die Möglichkeit zur Freistellung für das Projekt hatten. Die Teilnehmer waren zwischen 40 und 50 Jahren alt und hatten eine qualifizierte technische Ausbildung (Meister- und Technikerniveau). Unterschiede bestanden im Umfang ihres Erfahrungswissens und der jeweiligen Lerngeschwindigkeit.

Als Zeitbudget wurden für die Bearbeitung 200 Stunden je Teilnehmer vereinbart, die über einen größeren Zeitraum (ca. 6 Monate) erbracht werden sollten. Dadurch wurde ein agiles Lernprojekt mit einem Zeitaufwand von wöchentlich 6 Stunden im Team und einigen Stunden in Einzelarbeit realisierbar. Um den Teilnehmern die Möglichkeit zu geben, sich ausreichend auf das Lernprojekt zu konzentrieren, wurde ein Projektraum bereitgestellt und das Team bekam viel Aufmerksamkeit und Unterstützung durch die Vorgesetzten. Verabredet war, dass das Team einen Tag in der Woche von der sonstigen Arbeit freigestellt sein sollte, was allerdings im Arbeitsalltag nicht immer realisiert werden konnte.

\section{Projektmanagement in zwei Modulen}

Das agile Lernprojekt wurde in zwei Module aufgeteilt, die jeweils aus mehreren Etappen bestanden. Das erste Modul fand komplett in Teamarbeit statt und befasste sich mit den „Grundlagen des Projektmanagements“. Im zweiten Modul wurde in einer angeleiteten Anwendungsphase im Arbeitsprozess von jedem Teilnehmer ein aktuelles Projekt aus sei- 


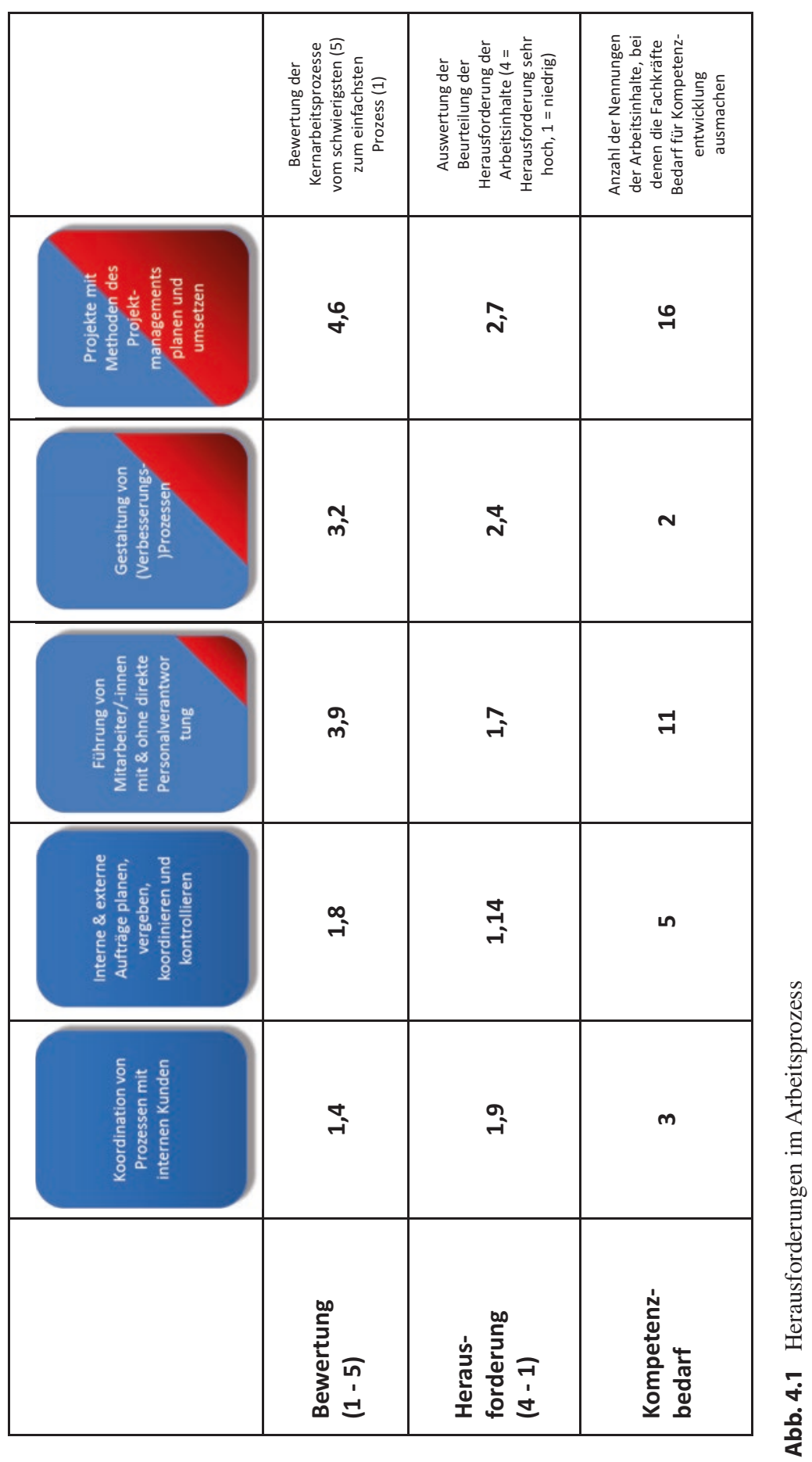




\section{Teamaufgabe \\ Aufgabe: Aufbau einer mit erneuerbarer Energie betriebenen Ladestation für E- Bikes auf dem Werksgelände \\ Akzeptanzkriterien: \\ - Technisch-inhaltliche Plausibilität \\ - Berücksichtigung von Randbedingungen wie Genehmigungsfähigkeit durch die Behörden und internen Prozessen \\ - Zu vertretbaren Kosten umsetzbar \\ - Tools des Projektmanagements wurden zweckmäßig eingesetzt \\ Beispiel einer Einzelaufgabe \\ Aufgabe: Verlegung des zentralen Daten-Verteilers für das gesamte Werksgelände in ein anderes Gebäude \\ Akzeptanzkriterien: \\ - ein unterbrechungsfreies Funktionieren des Daten-Verteilers während der ges- amten Verlegung ist gesichert \\ - alle externen und internen Vorschriften sind eingehalten (Baurecht, Brandschutz, Datensicherheit, Ausschreibungen etc.) \\ - Kosten sind ermittelt und das Budget ist genehmigt \\ - Planung des Umbaus abgeschlossen \\ - Tools des Projektmanagements wurden zweckmäßig eingesetzt}

Abb. 4.2 Teamaufgabe und ein Beispiel einer Einzelaufgabe

ner Fachabteilung bearbeitet (siehe Abb. 4.2). Der Zeitaufwand betrug einen Monat für das erste Modul und fünf Monate für das zweite Modul.

Die Lerninhalte bewegten sich auf akademischem Niveau. Das war für die Teilnehmenden schwierig, weil sie mit einer akademischer Arbeitsweise keine Erfahrungen hatten (hohe Abstraktion, Inhalte selbst erarbeiten etc.).

\subsection{Umsetzung}

Zu Beginn kamen die Teilnehmer durch die vielfältigen Anforderungen des Lernprojekts an die Grenzen ihrer Belastungsfähigkeit. Im Nachhinein beschrieben sie diese Phase als „Zustand maximaler Verwirrung“. Dieser Zustand ließ sich auf das komplexe Zusammenspiel aus fachlichen, persönlichen und organisatorischen Anforderungen zurückführen (Zitat: „Wir sind erst mal losgerannt und haben versucht, alle Probleme gleichzeitig zu lösen“). Außerdem hatten sie anfangs Schwierigkeiten, mit dem Umfang der zur Verfügung gestellten Unterlagen zurecht zu kommen.

Durch die enge Taktung des Austauschs zwischen Begleitern und Team konnte diese anfängliche Orientierungslosigkeit jedoch aufgefangen werden. Zusätzlich wurden die In- 
formationen zum Thema Projektmanagement ,portioniert“ und bei konkreten Herausforderungen unternehmensinterne Experten (erfahrene Projektmanager, Fachvorgesetzte und Prozessexperten) als „Interviewgäste“ eingeladen.

Dem Team gelang es nach und nach, sich die Struktur des Vorgehens im Projektmanagement zu erschließen und trotz der vielfältigen Anforderungen konstruktiv zusammen $\mathrm{zu}$ arbeiten. Dabei nutzten sie für die kurzfristige und formlose Kommunikation einen Messenger Dienst auf dem Smartphone (,Threema“). Zur Darstellung ihrer Abläufe und Zeitpläne eigneten sich die Teilnehmer MS Project an.

Im zweiten Modul wurde die Umsetzung der gelernten Grundlagen in realen Projekten aus dem eigenen Arbeitskontext angeleitet. Die Projekte in diesem zweiten Teil hatten fünf- bis sechsstellige Budgets und mussten z. T. aktuell durchgeführt werden. Eine mittlere oder gar mäßige Lösungsqualität war deshalb keine Option.

Die Umsetzung dieser Aufgaben beinhaltete auch den Aufbau eines Netzwerks an Ansprechpartnern innerhalb des Unternehmens. Jeweils an „Quality Gates“, die in firmeninternen Richtlinien des Projektmanagements vorgesehen sind, wurde der Arbeitsstand den direkten Fachvorgesetzten und bei der Abschlussveranstaltung auch dem Bereichsleiter vorgestellt. Von diesen wurden die Ergebnisse entweder abgenommen oder zur weiteren Bearbeitung zurückgegeben.

Schwierigkeiten im Ablauf gab es durch rotierende Urlaubszeiten und dadurch, dass ein Nachzügler in das Team aufgenommen wurde. Trotzdem fand eine gute Abstimmung untereinander statt und es gelang dem Team, die verschiedenen Aufgaben untereinander aufzuteilen. Anfangs hatten die Teammitglieder Hemmungen, die Ressource „Begleiter“ zu nutzen und um Erklärungen und Unterstützung zu bitten. Dies wurde zum Ende hin jedoch besser.

\section{Lernen heißt: Fehler machen dürfen}

Zentral für die Anwendungsphase war, dass Lernen und insbesondere selbstgesteuertes Lernen Spielräume braucht, um Lösungen auszuprobieren und auch Fehler machen zu dürfen. Da es sich jedoch um reale Projekte handelte, die unter einem starken Erfolgsdruck standen, musste hier in Abstimmung mit den Auftraggebern immer wieder Entfaltungsspielraum geschaffen werden, um beispielsweise alternative Lösungswege zu entwickeln. Der erste Impuls der Lerngruppe bestand trotzdem häufig darin, so schnell wie möglich eine Lösung zu finden und diese direkt umzusetzen. Die Teammitglieder konnten sich erst langsam von dieser ergebnisorientierten Haltung lösen, um die komplexen und neuen Problemstellungen vollständig zu erfassen und Lösungsalternativen zu prüfen.

Während der Reflexionsphasen gelang es den Mitarbeitenden anfangs nur schwer, sich von ihrer fachlichen Ebene zu lösen und auch ihre persönlichen Herausforderungen oder die Zusammenarbeit mit dem Team und den Begleitern zu hinterfragen. Als Unterstützung der Reflexion führten die Begleiter ein „Lern-Logbuch“ ein, in dem stichwortartig die Arbeits- und Erkenntnisschritte festgehalten werden sollten. Es wurde allerdings erst nach längeren Anlaufschwierigkeiten häufiger ausgefüllt. 
Insgesamt haben sich die Reflexionen nicht nur im Umfang, sondern auch in ihrer Tiefe über den Zeitraum des Teamprojektes stark verändert, zunehmend wurden auch soziale, didaktische und persönliche Themen angesprochen.

\subsection{Beobachtungen und Lessons Learned}

\section{Rückschau}

Inhaltlich wurden alle arbeitsplatzspezifischen Projekte nach Abschluss vom Auftraggeber und von den Fachvorgesetzten als fachlich einwandfrei bewertet. Außerdem wurde positiv hervorgehoben, dass die Teilnehmenden deutlich an Kompetenzen gewonnen hatten, sowohl im Projektmanagement und den innerbetrieblichen Begleitprozessen als auch im persönlichen Auftreten, etwa in Präsentationen. Dies wurde sicher zum Teil durch die durchgehend gute Unterstützung durch Kollegen und Vorgesetzte erreicht.

Insgesamt haben die Teilnehmer nicht nur eine hohe fachliche Qualität der technischen Ergebnisse erreicht, sondern auch Konzepte, Methoden und Tools des Projektmanagements kennengelernt und praxisgerecht angewandt. Darüber hinaus verstanden sie unternehmensinterne Prozesse besser, z. B. die Funktionen der Finanzplanung und der betrieblichen Bauabteilung. Dies war nur durch das Lernen an einem realen Projekt möglich und hätte in einem Planspiel oder einer Übungsaufgabe so nicht stattfinden können. Die Teilnehmenden wie die Vorgesetzten waren mit dem Lernprojekt sehr zufrieden und ein Teilnehmer fühlte sich durch das Projekt sogar ermutigt, ein berufsbegleitendes Masterstudium aufzunehmen.

\section{Was nehmen wir mit?}

- Die Zwänge der Alltagsarbeit lassen sich, insbesondere bei Lernenden mit einem wichtigen Verantwortungsbereich, nicht immer beiseite schieben. Es kam wiederholt vor, dass ein Teilnehmer kurzfristig eine Leistung nicht erbringen oder an einem Treffen nicht teilnehmen konnte, weil es einen dringenden betrieblichen Bedarf gab. An diesem Punkt ist ein Lernprojekt am Arbeitsplatz sicher auch im Nachteil gegenüber einem Lehrgang, der räumlich komplett getrennt stattfindet. Um so wichtiger ist es, belastbare Vereinbarungen zu treffen und im Alltag so weit wie möglich zu schützen.

- Reale Arbeitsaufgaben brauchen unbedingt ausgezeichnete Lösungen. Dies schafft ein Spannungsfeld, weil selbstgesteuertes Lernen immer auch Umwege und unzulängliche erste Ansätze beinhaltet. Grundsätzlich ist das agile Vorgehen mit kurzen Schritten hierbei hilfreich, weil Fehler und Unzulänglichkeiten schnell erkannt und korrigiert werden können. Gleichzeitig müssen Auftraggeber lernen, Spielräume für Lernprozesse zu ermöglichen.

- Beschäftigte, die keinen akademischen Hintergrund haben, sind oft wenig erfahren in selbstgesteuertem Lernen. Benötigtes Wissen gezielt aus umfangreichen und möglicherweise unstrukturierten Unterlagen oder einer breiten Recherche im Internet zu gewinnen, kann ihnen sehr schwerfallen. Lernbegleiter müssen dies berücksichtigen. Ein 
Weg dazu kann sein, Unterlagen in überschaubare Einheiten aufzuteilen, die in einem begrenzten Zeitraum, z. B. 30 Minuten, durchgearbeitet werden können und direkt hilfreich beim Bearbeiten der Lernaufgabe sind (vgl. Abschn. 14.3).

- Berufliche Praktiker sind oft besser im Lösen von Problemen als in der Reflexion. Diese braucht deshalb eine gezielte Anleitung und Unterstützung. Es kann helfen, wenn eine Reflexion auch außerhalb der Retrospektive unterstützt wird, z. B. durch ein „Lern-Logbuch“, in dem Fortschritte und Erkenntnisse festgehalten werden, oder durch ein „Lern-Portfolio“, in dem Zwischenergebnisse und sonstige Dokumente zusammengestellt sind.

\section{Literatur}

Ahrens, D., Dombrowski, T., Grantz, T., Heinze, H., Höhne, B., Kaßebaum, B., Kroll, S., Longmuß, J. \& Staden, C. (2018). Herausforderungen und Chancen betrieblicher Weiterbildung in digitalisierten Arbeitswelten. Abschlussbericht des Verbundprojekts: Berufliche Professionalität im produzierenden Gewerbe. Institut Technik und Bildung (ITB), Universität Bremen, ITBForschungsberichte 65

Open Access Dieses Kapitel wird unter der Creative Commons Namensnennung - Weitergabe unter gleichen Bedingungen 4.0 International Lizenz (http://creativecommons.org/licenses/ by-sa/4.0/deed.de) veröffentlicht, welche die Nutzung, Vervielfältigung, Bearbeitung, Verbreitung und Wiedergabe in jeglichem Medium und Format erlaubt, sofern Sie den/die ursprünglichen Autor(en) und die Quelle ordnungsgemäß nennen, einen Link zur Creative Commons Lizenz beifügen und angeben, ob Änderungen vorgenommen wurden. Wenn Sie das Buch oder Teile daraus remixen, verändern oder anderweitig direkt darauf aufbauen, dürfen Sie Ihre Beiträge nur unter derselben Lizenz wie das Original verbreiten.

Die in diesem Kapitel enthaltenen Bilder und sonstiges Drittmaterial unterliegen ebenfalls der genannten Creative Commons Lizenz, sofern sich aus der Abbildungslegende nichts anderes ergibt. Sofern das betreffende Material nicht unter der genannten Creative Commons Lizenz steht und die betreffende Handlung nicht nach gesetzlichen Vorschriften erlaubt ist, ist für die oben aufgeführten Weiterverwendungen des Materials die Einwilligung des jeweiligen Rechteinhabers einzuholen.

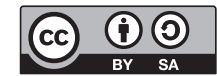

